\title{
Phenotypic characterization of X-linked hypophosphatemia in pediatric Spanish population
}

Enrique Rodríguez-Rubio ${ }^{1 *}$ (0), Helena Gil-Peña ${ }^{2}$, Sara Chocron ${ }^{3}$, Leire Madariaga ${ }^{4}$, Francisco de la Cerda-Ojeda ${ }^{5}$, Marta Fernández-Fernández ${ }^{6}$, Carmen de Lucas-Collantes ${ }^{7}$, Marta Gil ${ }^{8}$, María Isabel Luis-Yanes ${ }^{9}$, Inés Vergara ${ }^{10}$, Juan David González-Rodríguez ${ }^{11}$, Susana Ferrando ${ }^{12}$, Montserrat Antón-Gamero ${ }^{13}$, Marta Carrasco Hidalgo-Barquero ${ }^{14}$, Angustias Fernández-Escribano ${ }^{15}$, Mo Ángeles Fernández-Maseda ${ }^{16}$, Laura Espinosa ${ }^{17}$, Aniana Oliet ${ }^{18}$, Antonio Vicente ${ }^{19}$, Gema Ariceta $^{3}$ and Fernando Santos ${ }^{1,2}$ on behalf of RenalTubeGroup

\begin{abstract}
Background: $X$-linked hypophosphatemia $(X L H)$ is a hereditary rare disease caused by loss-of-function mutations in PHEX gene leading tohypophosphatemia and high renal loss of phosphate. Rickets and growth retardation are the major manifestations of XLH in children, but there is a broad phenotypic variability. Few publications have reported large series of patients. Current data on the clinical spectrum of the disease, the correlation with the underlying gene mutations, and the long-term outcome of patients on conventional treatment are needed, particularly because of the recent availability of new specific medications to treat XLH.

Results: The RenalTube database was used to retrospectively analyze 48 Spanish patients ( 15 men) from 39 different families, ranging from 3 months to 8 years and 2 months of age at the time of diagnosis (median age of 2.0 years), and with XLH confirmed by genetic analysis. Bone deformities, radiological signs of active rickets and growth retardation were the most common findings at diagnosis. Mean ( \pm SEM) height was $-1.89 \pm 0.19$ SDS and 55\% (22/40) of patients had height SDS below-2. All cases had hypophosphatemia, serum phosphate being $-2.81 \pm 0.11$ SDS. Clinical manifestations and severity of the disease were similar in both genders. No genotype-phenotype correlation was found. Conventional treatment did not attenuate growth retardation after a median follow up of 7.42 years $(\mathrm{IQR}=11.26 ; \mathrm{n}=26$ patients) and failed to normalize serum concentrations of phosphate. Eleven patients had mild hyperparathyroidism and 8 patients nephrocalcinosis.
\end{abstract}

Conclusions: This study shows that growth retardation and rickets were the most prevalent clinical manifestations at diagnosis in a large series of Spanish pediatric patients with XLH confirmed by mutations in the PHEX gene. Traditional treatment with phosphate and vitamin D supplements did not improve height or corrected hypophosphatemia and

\footnotetext{
*Correspondence: erodrr00@gmail.com

1 Pediatric Research, Medicine Department, University of Oviedo, Oviedo,

Spain

Full list of author information is available at the end of the article

The original online version of this article was updated to amend the

article's funding section.
}

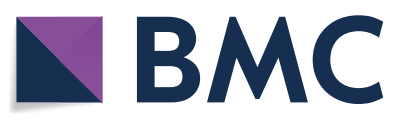
International License, which permits use, sharing, adaptation, distribution and reproduction in any medium or format, as long as you give appropriate credit to the original author(s) and the source, provide a link to the Creative Commons licence, and indicate if changes were made. The images or other third party material in this article are included in the article's Creative Commons licence, unless indicated otherwise in a credit line to the material. If material is not included in the article's Creative Commons licence and your intended use is not permitted by statutory regulation or exceeds the permitted use, you will need to obtain permission directly from the copyright holder. To view a copy of this licence, visit http://creativecommons.org/licenses/by/4.0/. The Creative Commons Public Domain Dedication waiver (http://creativecommons.org/publicdomain/zero/1.0/) applies to the data made available in this article, unless otherwise stated in a credit line to the data. 
was associated with a risk of hyperparathyroidism and nephrocalcinosis. The severity of the disease was similar in males and females.

Keywords: XLH, Inherited hypophosphatemia, Growth retardation, Bone deformities, Rickets

\section{Background}

X-linked hypophosphatemic rickets (XLH) (OMIM 307800) (ORPHA 89936) is the most common hereditary rickets $[1-5]$ with an estimated prevalence of 1:20,000 [6, 7]. It follows an X-linked dominant transmission [8]. The disease is caused by a defective function of PHEX gene [1, 9-13], leading to elevated circulating concentrations of fibroblast growth factor 23 (FGF23) [14], relatively low levels of 1,25 dihydroxyvitamin $\mathrm{D}\left[1,25(\mathrm{OH})_{2} \mathrm{D}\right]$, hyperphosphaturia secondary to decreased proximal tubular reabsorption of phosphate and hypophosphatemia $[8,10$, 15]. Classical, conventional treatment of XLH is based on the administration of phosphate supplements and 1-alpha hydroxylated derivates of vitamin D [16]. The wider availability of genetic studies and the recent development of an anti-FGF23 antibody, burosumab, as novel and promising therapy $[10,17]$ have resulted in a growing current interest for XLH.

We here report the clinical manifestations at diagnosis and follow-up of a large series of Spanish patients included in the online database RenalTube [18]. This study is justified at least by the following reasons: (1) $\mathrm{XLH}$ is a rare disease and few publications provide data on large series of patients; (2) XLH has a broad phenotypic variability and additional information is required to better characterize the clinical spectrum of the disease and to explain why the number of cases diagnosed usually does not correspond to the estimated prevalence of the disease; (3) It is important to share data of patients with genetically confirmed XLH in order to facilitate the finding of a potential phenotype-genotype correlation and to have current data that can be compared for the assessment of the new therapies.

\section{Results}

Forty-eight patients included in the RenalTube database with the diagnosis of XLH confirmed by defect-of-function variants found in the PHEX gene were analyzed. All variants had been identified as pathogenic. Sixteen patients (33\%) had variants with strong evidence of pathogenicity (nonsense, frameshift, deletions) while the other $32(67 \%)$ harbored variants with very strong evidence of pathogenicity (SNPs). Demographic and genetic data from patients are shown in Table 1. Patients were from 39 families and were being followed in pediatric nephrology units of 17 Spanish hospitals (Fig. 1). Fifteen patients were males and 33 females. Median age at diagnosis was 2.0 (IQR 2.6) years and the age ranged from 3 months to 8 years 2 months.

Presenting manifestations are shown in Table 2 for each patient. Bone deformities and radiological signs of active rickets were the most frequent findings leading to diagnosis. Ten patients were diagnosed because of family screening. Age at diagnosis of these patients was no different from that of the rest of the series as it ranged from 0.5 to 8 years with a median age of 1.04 years.

Twenty-two out of 40 patients (55\%) in whom the height was registered presented growth retardation (height $\leq 2$ SDS). Patients' height $(\mathrm{X} \pm$ SEM) was $-1.89 \pm 0.19$ SDS $(\mathrm{n}=40)$ (Fig. 2). In $87 \%(35 / 40)$ the height was below the 50 th percentile. Weight was $-0.88 \pm 0.14$ SDS $(n=41)$ and body mass index $0.2 \pm 0.15$ SDS $(\mathrm{n}=40)$.

Biochemical findings at diagnosis are shown in Table 3. Mean values $( \pm$ SEM) of available data were serum phosphate $2.7 \pm 0.1 \mathrm{mg} / \mathrm{dl} ;-2.81 \pm 0.11$ SDS, $(\mathrm{n}=41)$, alkaline phosphatase $(892 \pm 84 \mathrm{mU} / \mathrm{ml})(\mathrm{n}=39)$, $1,25(\mathrm{OH})_{2} \mathrm{D} 62 \pm 7 \mathrm{pg} / \mathrm{ml}(\mathrm{n}=34)$, parathyroid hormone $(\mathrm{PTH}) 70 \pm 7 \mathrm{pg} / \mathrm{ml}(\mathrm{n}=33)$, and tubular phosphate reabsorption (TPR) $69 \pm 4 \%(\mathrm{n}=26)$.

No differences were found between males and females for clinical manifestations, growth impairment or biochemical data at diagnosis. Likewise, no genotype-phenotype correlation was found. Actually, even patients within the same family presented different severity of clinical and biochemical manifestations.

Growth and biochemical variables of 26 patients after a median follow uptime of 7.42 years $(\mathrm{IQR}=11.26)$ are shown in Table 4, Figs. 3 and 4. Anthropometric data were $-1.94 \pm 0.16$ SDS for height $(n=24),-0.82 \pm 0.10$ SDS for weight $(\mathrm{n}=22)$ and $0.14 \pm 0.19$ SDS for BMI $(n=22)$. Comparison of data from patients with information both at diagnosis and last follow-up showed mean variations of $0.13 \pm 0.23$ SDS for height $(p>0.05)$ $(\mathrm{n}=20), 0.35 \pm 0.14$ SDS for weight $(\mathrm{p}=0.02)(\mathrm{n}=20)$ and $0.13 \pm 0.20$ SDS for BMI $(\mathrm{p}>0.05)(\mathrm{n}=20)$.

Mean SDS for serum phosphate was $-2.72 \pm 0.20$ $(\mathrm{n}=25)$. Alkaline phosphatases, $1,25(\mathrm{OH})_{2} \mathrm{D}$ and PTH levels were $525 \pm 82 \mathrm{mU} / \mathrm{ml}(\mathrm{n}=14), 53 \pm 7 \mathrm{pg} / \mathrm{ml}$ $(\mathrm{n}=15)$ and $68 \pm 8 \mathrm{pg} / \mathrm{ml}(\mathrm{n}=26)$ respectively. Mean tubular phosphate reabsorption was $65 \pm 3 \%(n=22)$. Comparison between diagnosis and last follow-up data revealed a variation of $0.20 \pm 0.28$ SDS for serum phosphate $(\mathrm{p}>0.05)(\mathrm{n}=20), 5 \pm 7 \mathrm{pg} / \mathrm{ml}$ for $1,25(\mathrm{OH})_{2} \mathrm{D}$ $(\mathrm{p}>0.05) \quad(\mathrm{n}=10), \quad 0 \pm 11 \mathrm{pg} / \mathrm{ml}$ for PTH $(\mathrm{p}>0.05)$ 
Table 1 Demographic and genetic data of 48 patients belonging to 39 families (Roman number indicates family)

\begin{tabular}{|c|c|c|c|c|c|}
\hline Patient & Relationship & Sex & cDNA mutation & Protein mutation & Variant type \\
\hline 1.1 & Index & $\mathrm{F}$ & c.758_759delTT & p.F26Cfs & $P(P \vee S 1)$ \\
\hline 1.2 & Sister & $\mathrm{F}$ & c.758_759delTT & p.F26Cfs & $\mathrm{P}$ (PVS1) \\
\hline II.1 & Index & $\mathrm{F}$ & c.2223_2224delAC & p.A514Afs516X & $P(P \vee S 1)$ \\
\hline III.1 & Index & $\mathrm{F}$ & c.1578_1579delAA & p.K299Nfs304X & $P(P \vee S 1)$ \\
\hline IV.1 & Index & M & c.893A $>T$ & p.N71I & $P(P S 1)$ \\
\hline IV.2 & Brother & M & C.893A $>T$ & p.N71I & $P(P S 1)$ \\
\hline V.1 & Index & $\mathrm{F}$ & c. $2633 G>C$ & p.R651P & $P(P S 1)$ \\
\hline VI.1 & Index & $\mathrm{F}$ & c. $1885 C>T$ & p.Q402X & $P(P \vee S 1)$ \\
\hline VII.1 & Index & M & c.?-2664dup2949-? & Splice region variant & $P(P \vee S 1)$ \\
\hline VII.2 & Brother & M & c.?-2664dup2949-? & Splice region variant & $P(P \vee S 1)$ \\
\hline VII.3 & Daughter & $\mathrm{F}$ & c.?-2664dup2949-? & Splice region variant & $P(P \vee S 1)$ \\
\hline VIII.4 & Daughter & $\mathrm{F}$ & c.?-2664dup2949-? & Splice region variant & $P(P \vee S 1)$ \\
\hline VIII.1 & Index & $\mathrm{F}$ & c.886insT & p.K69X & $P(P \vee S 1)$ \\
\hline IX.1 & Index & $\mathrm{F}$ & c. $2048 \mathrm{G}>\mathrm{A}$ & p.W456X & $P(P \vee S 1)$ \\
\hline$X .1$ & Index & $\mathrm{F}$ & g.22099152G>T & Splice region variant & $P(P \vee S 1)$ \\
\hline XI.1 & Index & M & c.2648_?del & p.A656_?del & $P(P \vee S 1)$ \\
\hline XII.1 & Index & $\mathrm{F}$ & c.2327_?del & p.R549_?del & $P(P \vee S 1)$ \\
\hline XIII.1 & Index & $\mathrm{F}$ & g.22168393_delA & Splice region variant & $P(P \vee S 1)$ \\
\hline XIV.1 & Index & $\mathrm{F}$ & c. $1552 C>T$ & p.R291X & $P(P \vee S 1)$ \\
\hline$X V .1$ & Index & $\mathrm{F}$ & g. $22190503 \mathrm{G}>\mathrm{A}$ & Splice region variant & $P(P \vee S 1)$ \\
\hline$X V I .1$ & Index & $\mathrm{F}$ & c.889_893delGTAAA & p.V70Sfs77X & $P(P \vee S 1)$ \\
\hline XVII.1 & Index & $\mathrm{F}$ & c.2086_?del & p.A469_?del & $P(P \vee S 1)$ \\
\hline XVIII.1 & Index & $\mathrm{F}$ & C. $1180 \mathrm{~T}>\mathrm{C}$ & p.W167R & $P(P S 1)$ \\
\hline XIX.1 & Index & $\mathrm{F}$ & $c .2282 C>T$ & p.P534L & $P(P S 1)$ \\
\hline$X X .1$ & Index & $\mathrm{F}$ & $c .2416 \mathrm{G}>\mathrm{A}$ & p.G579R & $P(P S 1)$ \\
\hline XXI.1 & Index & $\mathrm{F}$ & c. $2920 C>T$ & p.R747X & $P(P \vee S 1)$ \\
\hline XXII.1 & Index & $\mathrm{F}$ & c. $2920 C>T$ & p.R747X & $P(P \vee S 1)$ \\
\hline XXIII.1 & Index & M & c.1152delA & p.L157Lfs220X & $P(P \vee S 1)$ \\
\hline XXIV.1 & Index & $\mathrm{F}$ & c.1572C>A,c.1580_1582delTGA & p.Y297X & $P(P \vee S 1)$ \\
\hline$X X V .1$ & Index & $\mathrm{F}$ & g. $22076478 \mathrm{~A}>\mathrm{T}$ & Splice region variant & $P(P \vee S 1)$ \\
\hline XXVI.1 & Index & $\mathrm{F}$ & g.22190507G>A & Splice region variant & $P(P \vee S 1)$ \\
\hline XXVII.1 & Index & $\mathrm{F}$ & c.889_893delGTAAA & p.V70Sfs77X & $P(P \vee S 1)$ \\
\hline$X X V I I .2$ & Father & M & c.889_893delGTAAA & p.V70Sfs77X & $P(P \vee S 1)$ \\
\hline XXVIII.1 & Index & M & c. $2879 \mathrm{G}>\mathrm{T}$ & p.C733F & $P(P S 1)$ \\
\hline$X X V I I I .2$ & Sister & $\mathrm{F}$ & c. $2879 \mathrm{G}>\mathrm{T}$ & p.C733F & $P(P S 1)$ \\
\hline XXIX.1 & Index & $\mathrm{F}$ & c. $2642 T>C$ & p.F654S & $P(P S 1)$ \\
\hline$X X X .1$ & Index & M & c. $2387 \mathrm{~T}>\mathrm{G}$ & p.L569R & $P(P S 1)$ \\
\hline$X X X .2$ & Mother & $\mathrm{F}$ & c. $2387 \mathrm{~T}>\mathrm{G}$ & p.L569R & $P(P S 1)$ \\
\hline XXXI.1 & Index & $\mathrm{F}$ & c. $2085 G>C$ & p.K468N & $P(P S 1)$ \\
\hline XXXII.1 & Index & M & c. $2282 C>T$ & p.P534L & $P(P S 1)$ \\
\hline XXXIII.1 & Index & M & g. $22033125 T>G$ & Splice region variant & $P(P V S 1)$ \\
\hline XXXIV.1 & Index & M & $c .2380 C>T$ & p.R567X & $P(P \vee S 1)$ \\
\hline$X X X V .1$ & Index & M & c. $2005 \mathrm{G}>\mathrm{T}$ & p.V442P & $P(P S 1)$ \\
\hline$X X X V .2$ & Mother & $\mathrm{F}$ & c. $2005 \mathrm{G}>\mathrm{T}$ & p.V442P & $P(P S 1)$ \\
\hline XXXVI.1 & Index & $\mathrm{F}$ & c. $2416 \mathrm{G}>\mathrm{A}$ & G579R & $P(P S 1)$ \\
\hline XXXVIII.1 & Index & M & g.22099152G>A & Splice region variant & $P(P V S 1)$ \\
\hline XXXVIII.1 & Index & $\mathrm{F}$ & c.2617delG & p.D646lfs & $P(P V S 1)$ \\
\hline XXXIX.1 & Index & M & c.1363_1364delTC & p.S228Pfs236X & $P(P V S 1)$ \\
\hline
\end{tabular}

F: female, M: male

$P:$ pathogenic 
Table 1 (continued)

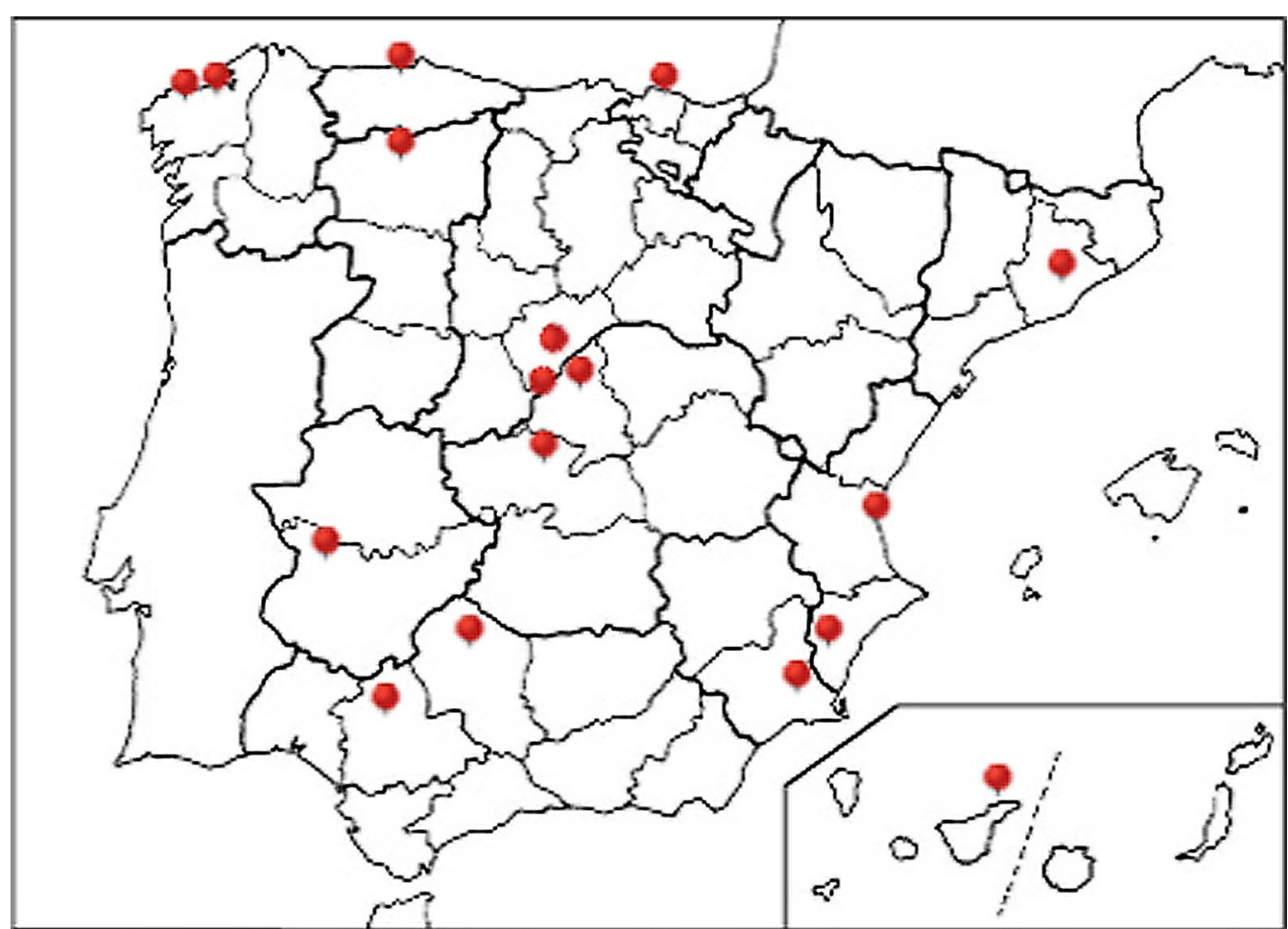

Fig. 1 Geographical distribution of the Spanish hospitals participating in the study

$(\mathrm{n}=16)$ and $-7 \pm 8$ for tubular phosphate reabsorption $(\mathrm{p}>0.05)(\mathrm{n}=11)$.

Eight out of 24 patients with renal ultrasounds at last follow-up presented nephrocalcinosis (Table 5).

\section{Discussion}

This study provides a current description of the phenotypic characteristics of a large cohort of Caucasian pediatric patients with XLH genetically confirmed. The sample is a broad representation of the Spanish children with XLH, coming from several hospitals scattered through the country and provides data at diagnosis and after a median follow-up of 7.42 years. The study confirms that growth retardation, bone deformities and active lesions of rickets are the main presenting manifestations of the disease, within a wide spectrum of symptoms. No significant differences were found between males and females as for the severity of the disease. It is of interest that a broad spectrum of PHEX gene variants, all of them already described as pathogenic, was found and no mutation was specifically prevalent in Spanish population. All variants were classified as pathogenic according to the American College of Medical Genetics and Genomics consensus [19]. There was a high phenotypical variability even among family members harboring the same mutations, suggesting that other genes and environmental factors may affect the severity of XLH, as reported by other authors [20,21].

In addition, the study shows that conventional treatment with phosphate supplements and vitamin D metabolites does not lead to persistent correction of hypophosphatemia or reduction of renal wasting of phosphate and does not modify the circulating levels of calcitriol. Unfortunately, this study does not provide information on circulating FGF23 levels, given its retrospective design. At the last follow-up visit, 11 out of 25 patients had serum PTH values mildly elevated. In $\mathrm{XLH}$, development of hyperparathyroidism is thought to be related with the pharmacological administration of phosphate [22]. Eight patients developed nephrocalcinosis during the follow-up period, a finding linked to the 
Table 2 Clinical manifestations at diagnosis

\begin{tabular}{|c|c|c|c|c|c|}
\hline Patient & Age at diagnosis & Bone deformities & Active rickets & $\begin{array}{l}\text { Longitudinal growth retardation } \\
\text { ( } \leq 2 \text { SDS) }\end{array}$ & Dental problems \\
\hline 1.1 & $3 m$ & No & - & No & - \\
\hline 1.2 & $1 y$ & Yes & - & Yes & - \\
\hline II.1 & 1 y $5 \mathrm{~m}$ & Yes & Yes & Yes & No \\
\hline III.1 & 1 y $6 \mathrm{~m}$ & - & Yes & Yes & Yes \\
\hline IV.1 & 1 y $4 \mathrm{~m}$ & No & No & Yes & Yes \\
\hline IV.2 & $4 y$ & No & No & No & No \\
\hline V.1 & $5 y$ & Yes & Yes & Yes & No \\
\hline Vl.1 & $2 y$ & Yes & No & No & No \\
\hline VII.1 & $8 y$ & - & - & - & - \\
\hline VIII.2 & $2 y$ & - & Yes & - & No \\
\hline VIII.3 & $11 \mathrm{~m}$ & - & Yes & Yes & No \\
\hline VIII.4 & $1 y$ & - & - & Yes & - \\
\hline VIII.1 & $4 y$ & Yes & Yes & No & No \\
\hline IX.1 & $5 y$ & Yes & Yes & No & No \\
\hline$X .1$ & $5 y$ & Yes & Yes & Yes & No \\
\hline XI.1 & 2 y $3 \mathrm{~m}$ & Yes & Yes & Yes & No \\
\hline XII.1 & $2 y$ & Yes & Yes & - & - \\
\hline XIII.1 & $5 y$ & - & Yes & - & - \\
\hline XIV.1 & $9 m$ & Yes & Yes & No & No \\
\hline$X V .1$ & 1 y $1 \mathrm{~m}$ & Yes & Yes & Yes & - \\
\hline XVI.1 & $4 y$ & Yes & - & Yes & No \\
\hline XVII.1 & $5 y$ & - & - & Yes & - \\
\hline XVIII.1 & $2 y$ & Yes & Yes & Yes & No \\
\hline XIX.1 & $7 \mathrm{~m}$ & Yes & Yes & - & No \\
\hline$X X .1$ & $2 \mathrm{y} 1 \mathrm{~m}$ & Yes & - & Yes & - \\
\hline XXI.1 & 4 y $5 \mathrm{~m}$ & Yes & Yes & No & No \\
\hline XXII.1 & $4 y$ & Yes & Yes & Yes & No \\
\hline XIII.1 & $2 y$ & Yes & Yes & No & No \\
\hline XXIV.1 & $3 y$ & Yes & - & No & - \\
\hline$X X V .1$ & 1 y 6 & Yes & Yes & No & No \\
\hline XXVI.1 & $4 y$ & Yes & Yes & Yes & No \\
\hline XXVII.1 & $6 \mathrm{~m}$ & Yes & Yes & No & No \\
\hline$X X V I I .2$ & $8 y$ & - & Yes & - & No \\
\hline XXVIII.1 & 1 y $6 \mathrm{~m}$ & Yes & - & No & - \\
\hline XXVIII.2 & $6 \mathrm{~m}$ & Yes & Yes & No & No \\
\hline XXIX.1 & 1 y $9 \mathrm{~m}$ & Yes & Yes & Yes & No \\
\hline XXX.1 & $1 \mathrm{y} 1 \mathrm{~m}$ & Yes & Yes & Yes & - \\
\hline$X X X .2$ & 1 y $6 \mathrm{~m}$ & - & Yes & - & No \\
\hline XXXI.1 & 8 y2 m & Yes & Yes & Yes & No \\
\hline XXXII.1 & 1 y $6 \mathrm{~m}$ & Yes & Yes & No & No \\
\hline XXXIII.1 & $5 y$ & - & - & Yes & - \\
\hline XXXIV.1 & $2 y 2 \mathrm{~m}$ & Yes & Yes & No & No \\
\hline$X X X V .1$ & $7 \mathrm{~m}$ & Yes & Yes & No & - \\
\hline$X X X V .2$ & 2 y $6 \mathrm{~m}$ & Yes & Yes & Yes & - \\
\hline XXXVI.1 & $6 \mathrm{~m}$ & Yes & Yes & No & No \\
\hline XXXVII.1 & 1 y $9 \mathrm{~m}$ & Yes & Yes & Yes & Yes \\
\hline XXXVIII.1 & 1 y $6 \mathrm{~m}$ & Yes & Yes & No & No \\
\hline XXXIX.1 & $2 y 2 \mathrm{~m}$ & Yes & Yes & - & No \\
\hline \multirow{2}{*}{\multicolumn{2}{|c|}{ Percentage of patients }} & $P / A / U$ & $\mathrm{P} / \mathrm{A} / \mathrm{U}$ & $P / A / U$ & $P / A / U$ \\
\hline & & $73 / 6 / 21$ & $73 / 6 / 21$ & $46 / 38 / 17$ & $6 / 63 / 31$ \\
\hline
\end{tabular}


Table 2 (continued)

m: month, y: year

SDS: standard deviation score. Dash: information in this field was missing from the database

P/A/U: present/absent/unreported

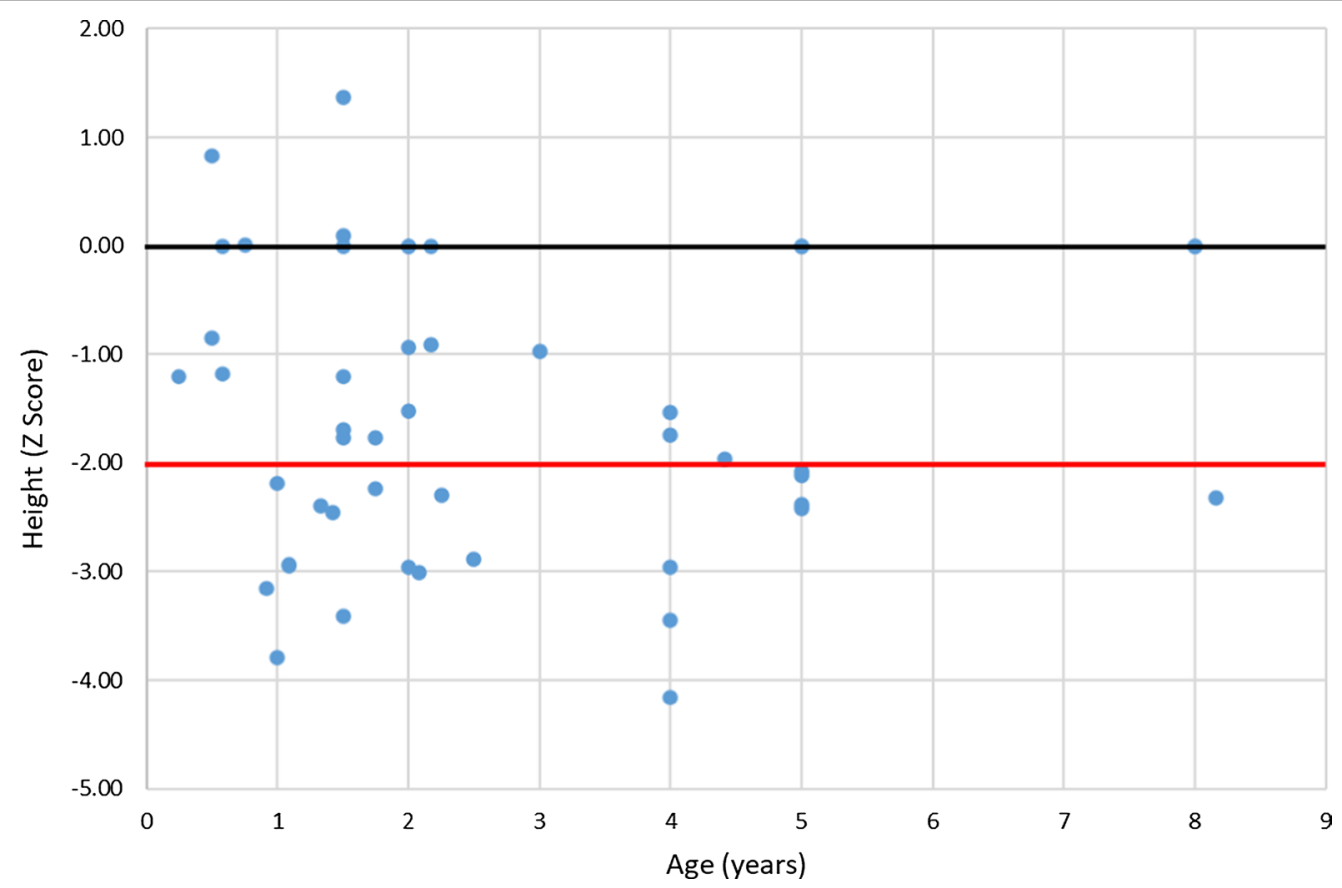

Fig. 2 Height at diagnosis $(n=40)$. Black line: 0 SD; red line: -2 SD

administration of phosphate and vitamin D that usually does not result in subsequent clinical complications [16].

Though conventional treatment has been described to heal active signs of rickets and may improve bone deformities [23], this study confirms that it does not lead to catch-up growth. Mean height $\mathrm{Z}$ score of the group of patients remained low, -1.89 at diagnosis versus -1.94 at the last visit, although Fig. 3 indicates that the individual patients' response varied from marked improvement to worsening of growth impairment. Two patients, VII.3 and XV.1, transiently received growth hormone treatment and their heights improved +2.19 and +0.66 SDS, respectively. It is of note that 4 out of 16 patients had BMI greater than +1.00 SDS at the last follow-up visit. This percentage of $25 \%$ corresponds to the normal distribution of reference population and it indicates that tendency to overweight and obesity was not found in the group of XLH patients here reported, unlike other series that have recently drawn attention to these complications likely related with the sedentary life and restricted mobility of these patients [11]. In this regard, a slight but significant increase in weight was found during the follow-up period in our series.
Our study presents methodological limitations inherent to the retrospective analysis and to the fact that patients' information was extracted from a database in which some data were missing and cannot be recovered. It is also of note the lack of information on the degree of adherence to medication of each patient as well as the different monitoring protocols among the participating centers. However, it is an observational clinical study describing a large cohort of Spanish pediatric patients with genetically confirmed XLH and it provides current and interesting information on the clinical and biochemical features of the disease, at diagnosis and follow-up after conventional treatment. Our findings could be used as reference for further studies using burosumab treatment.

\section{Conclusions}

This study confirms that growth retardation and rickets were the most prevalent clinical manifestations at diagnosis in a large series of Spanish pediatric patients with XLH confirmed by identification of pathogenic variants in the PHEX gene. Traditional treatment with phosphate supplements and calcitriol did not improve height or corrected hypophosphatemia and was associated with a risk 
Table 3 Biochemical manifestations at diagnosis

\begin{tabular}{|c|c|c|c|c|c|c|}
\hline \multirow[t]{2}{*}{ Patient } & \multicolumn{2}{|c|}{ Serum phosphate } & \multirow{2}{*}{$\begin{array}{l}\text { Serum alkaline phosphatases } \\
(\mathrm{mU} / \mathrm{ml})\end{array}$} & \multirow{2}{*}{$\begin{array}{l}\text { Serum 1,25(OH) })_{2} \mathrm{D}(\mathrm{pg} / \\
\mathrm{ml})\end{array}$} & \multirow[t]{2}{*}{ Serum intact PTH (pg/ml) } & \multirow[t]{2}{*}{ TPR (\%) } \\
\hline & $\mathrm{mg} / \mathrm{dl}$ & SDS & & & & \\
\hline 1.1 & 3.4 & -2.21 & 1230 & 87 & - & - \\
\hline 1.2 & 3.8 & -1.05 & 1916 & 76 & - & - \\
\hline II.1 & 2.6 & -2.68 & 695 & 57 & 81 & 79 \\
\hline III.1 & 2.1 & -3.36 & 1646 & 16 & 68 & 32 \\
\hline IV.1 & 2.8 & -2.41 & 378 & 73 & 29 & 78 \\
\hline IV.2 & 3.0 & -2.44 & 232 & 40 & 43 & 82 \\
\hline V.1 & 2.1 & -3.99 & 1513 & 147 & 78 & 85 \\
\hline Vl.1 & 2.6 & -2.68 & 525 & 33 & 64 & 78 \\
\hline VII.1 & - & - & - & - & - & - \\
\hline VII.2 & 2.4 & -2.95 & 516 & - & - & - \\
\hline VII.3 & - & - & 991 & 28 & 30 & - \\
\hline VIII.4 & - & - & - & - & - & - \\
\hline VIII.1 & 3.1 & -2.27 & 598 & 55 & 39 & 38 \\
\hline IX.1 & 3.0 & -2.44 & 1824 & 47 & 59 & 67 \\
\hline$X .1$ & 2.9 & -2.61 & 639 & 33 & 136 & 74 \\
\hline XI.1 & 2.3 & -3.09 & - & - & 58 & - \\
\hline XII.1 & 2.7 & -2.54 & - & - & 48 & - \\
\hline XIII.1 & - & - & - & - & - & - \\
\hline XIV.1 & 3.3 & -2.34 & 892 & 64 & 65 & 75 \\
\hline$X V .1$ & 2.0 & -3.49 & 704 & 20 & - & 39 \\
\hline XVI.1 & 2.4 & -3.47 & 571 & 108 & 71 & 26 \\
\hline XVIII.1 & 2.5 & -3.30 & - & 28 & 30 & - \\
\hline XVIII.1 & 2.3 & -3.09 & 1864 & 31 & 64 & 73 \\
\hline XIX.1 & - & - & - & - & - & - \\
\hline$X X .1$ & 2.9 & -2.27 & 446 & 88 & 116 & 93 \\
\hline XXI.1 & 3.1 & -2.27 & 470 & 56 & 49 & 86 \\
\hline XXII.1 & 2.8 & -2.78 & 697 & 74 & 32 & 76 \\
\hline XXIII.1 & 2.1 & -3.36 & 733 & - & 68 & 82 \\
\hline XXIV.1 & 2.2 & -3.22 & 514 & 61 & 78 & 58 \\
\hline XXV.1 & 2.9 & -2.27 & 1940 & 40 & 57 & 58 \\
\hline XXVI.1 & 2.3 & -3.64 & 423 & - & - & 82 \\
\hline XXVII.1 & 2.9 & -2.86 & 432 & 31 & 23 & - \\
\hline$X X V \| I .2$ & - & - & - & - & - & - \\
\hline XXVIII.1 & 3.0 & -2.14 & 236 & 78 & 54 & - \\
\hline$X X V \mid I I .2$ & 3.3 & -2.34 & 426 & 171 & 57 & 90 \\
\hline XXIX.1 & 1.8 & -3.77 & 1555 & - & - & - \\
\hline XXX.1 & 3.1 & -2.00 & 829 & 183 & 101 & 88 \\
\hline$X X X .2$ & 2.2 & -3.22 & 158 & 25 & - & - \\
\hline XXXI.1 & - & - & - & - & - & - \\
\hline XXXII.1 & 2.8 & -2.41 & 692 & 41 & 111 & - \\
\hline XXXIII.1 & 2.1 & -3.99 & - & - & 76 & 54 \\
\hline XXXIV.1 & 2.3 & -3.09 & 856 & 64 & 101 & 46 \\
\hline XXXV.1 & 3.5 & -2.08 & 916 & - & 227 & 82 \\
\hline$X X X V .2$ & 2.2 & -3.22 & 1620 & - & - & 70 \\
\hline XXXVI.1 & 2.8 & -2.41 & 1093 & 65 & 66 & - \\
\hline XXXVII.1 & 2.4 & -2.95 & 1527 & 46 & 94 & - \\
\hline XXXVIII.1 & 2.4 & -2.95 & 856 & 40 & 58 & - \\
\hline XXXIX.1 & 3.0 & -2.14 & 759 & 51 & 43 & - \\
\hline
\end{tabular}

1,25(OH) $)_{2}$ D: 1,25 dihydroxyvitamin D. PTH: Parathyroid hormone

TPR: tubular phosphate reabsorption. Dash: information in this field was missing from the database 
Table 4 Biochemical manifestations at last follow up

\begin{tabular}{|c|c|c|c|c|c|c|c|}
\hline \multirow[t]{2}{*}{ Patient } & \multirow[t]{2}{*}{ Follow-up Time } & \multicolumn{2}{|c|}{ Serum phosphate } & \multirow{2}{*}{$\begin{array}{l}\text { Serum alkaline } \\
\text { phosphatases }(\mathrm{mU} / \mathrm{ml})\end{array}$} & \multirow{2}{*}{$\begin{array}{l}\text { Serum 1,25(OH) })_{2} \mathrm{D} \\
(\mathrm{pg} / \mathrm{ml})\end{array}$} & \multirow{2}{*}{$\begin{array}{l}\text { Serum intact PTH } \\
(\mathrm{pg} / \mathrm{ml})\end{array}$} & \multirow[t]{2}{*}{ TPR (\%) } \\
\hline & & $\mathrm{mg} / \mathrm{dl}$ & SD (Z score) & & & & \\
\hline 1.1 & 7 y $2 m$ & 2.9 & -2.07 & 787 & - & 30 & 72 \\
\hline 1.2 & 10 y $1 \mathrm{~m}$ & 2.7 & -2.90 & 1035 & - & 35 & 50 \\
\hline II.1 & 1 y $1 \mathrm{~m}$ & 3.8 & -1.05 & - & - & 34 & 70 \\
\hline |II.1 & - & - & - & - & - & - & - \\
\hline IV.1 & $7 y 6 m$ & 3.0 & -1.91 & 231 & 78 & 32 & 60 \\
\hline IV.2 & $7 y 6 m$ & 2.6 & -3.06 & 266 & 73 & 44 & 48 \\
\hline V.1 & - & - & - & - & - & - & - \\
\hline VI.1 & - & - & - & - & - & - & - \\
\hline VII.1 & - & - & - & - & - & - & - \\
\hline VII.2 & - & - & - & - & - & - & - \\
\hline VIII.3 & 8 y $4 \mathrm{~m}$ & 1.9 & -3.69 & - & - & 77 & 42 \\
\hline VII.4 & 8 y $3 \mathrm{~m}$ & 1.8 & -3.85 & 665 & - & 108 & 73 \\
\hline VIII.1 & $2 y 7 m$ & 2.4 & -3.47 & 868 & 15 & 84 & 81 \\
\hline IX.1 & - & - & - & - & - & - & - \\
\hline$X .1$ & 7 y $5 \mathrm{~m}$ & 3.0 & -2.41 & 962 & 50 & 93 & 85 \\
\hline XI.1 & 5 y $11 \mathrm{~m}$ & 2.8 & -2.78 & 632 & 45 & 49 & 42 \\
\hline XII.1 & 11 y $5 \mathrm{~m}$ & 2.8 & -2.23 & 636 & - & 112 & 86 \\
\hline XIII.1 & 17 y $6 \mathrm{~m}$ & 1.7 & -4.22 & - & 29 & 86 & 64 \\
\hline XIV.1 & $2 \mathrm{y} 1 \mathrm{~m}$ & 2.8 & -2.41 & - & 96 & 20 & 70 \\
\hline$X V .1$ & 25 y $5 \mathrm{~m}$ & 2.0 & -3.55 & - & 20 & 75 & 45 \\
\hline$X V I .1$ & - & - & - & - & - & - & - \\
\hline XVII.1 & 18 y $8 \mathrm{~m}$ & 1.7 & -4.22 & 120 & 32 & 99 & 42 \\
\hline XVIII.1 & - & - & - & - & - & - & - \\
\hline XIX.1 & 26 y $2 \mathrm{~m}$ & 2.4 & -2.66 & - & - & 200 & - \\
\hline$X X .1$ & 1 y $9 \mathrm{~m}$ & 3.3 & -1.73 & 312 & 72 & 53 & 45 \\
\hline XXI.1 & 3 y $5 \mathrm{~m}$ & 2.6 & -2.56 & 348 & 43 & 26 & 53 \\
\hline XXII.1 & - & - & - & - & - & - & - \\
\hline XXIII.1 & 2 y $10 \mathrm{~m}$ & 3.0 & -2.44 & - & 29 & 28 & - \\
\hline XXIV.1 & - & - & - & - & - & - & - \\
\hline XXV.1 & $16 y$ & 1.9 & -3.77 & - & - & 37 & - \\
\hline XXVI.1 & 2 y $4 \mathrm{~m}$ & 2.8 & -2.78 & 240 & 86 & 25 & 70 \\
\hline XXVII.1 & 1 y $10 \mathrm{~m}$ & 2.8 & -2.41 & - & 58 & 49 & 76 \\
\hline$X X V I I .2$ & 37 y $4 \mathrm{~m}$ & 2.3 & -2.88 & - & - & 117 & 80 \\
\hline XXVIII.1 & - & - & - & - & - & - & - \\
\hline$X X V I I I .2$ & - & - & - & - & - & - & - \\
\hline XXIX.1 & 1 y $10 \mathrm{~m}$ & 4.8 & 0.31 & 244 & 68 & 73 & 89 \\
\hline XXX.1 & - & - & - & - & - & - & - \\
\hline$X X X .2$ & - & - & - & - & - & - & - \\
\hline XXXI.1 & - & - & - & - & - & - & - \\
\hline XXXII.1 & - & - & - & - & - & - & - \\
\hline XXXIII.1 & $4 y$ & 2.1 & -3.36 & - & - & 114 & 76 \\
\hline XXXIV.1 & - & - & - & - & - & - & - \\
\hline XXXV.1 & - & - & - & - & - & - & - \\
\hline$X X X V .2$ & - & - & - & - & - & - & - \\
\hline XXXVI.1 & - & - & - & - & - & - & - \\
\hline XXXVII.1 & - & - & - & - & - & - & - \\
\hline XXXVIII.1 & - & - & - & - & - & - & - \\
\hline XXXIX.1 & - & - & - & - & - & - & - \\
\hline
\end{tabular}


Table 4 (continued)

$1,25(\mathrm{OH})_{2} \mathrm{D}: 1,25$ dihydroxyvitamin $\mathrm{D}$

m: month, y: year

PTH: parathyroid hormone

TPR: tubular phosphate reabsorption. Dash: information in this field was missing from the database
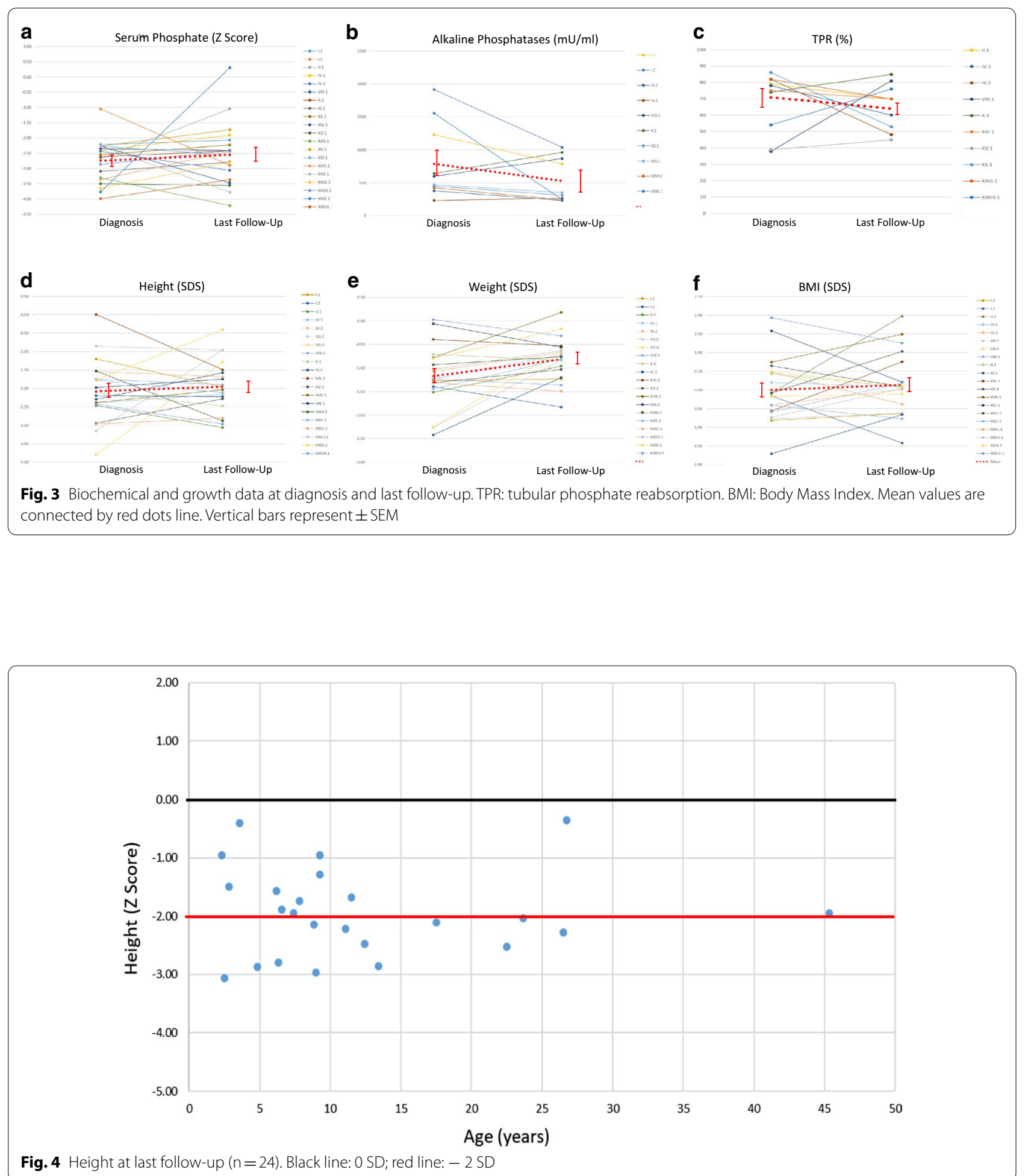

Fig. 4 Height at last follow-up $(n=24)$. Black line: 0 SD; red line: - 2 SD 
Table 5 Treatment and clinical data at last follow-up

\begin{tabular}{|c|c|c|c|}
\hline Patient & $\begin{array}{l}\text { Phosphorus element } \\
\text { dose (mg/kg/day) }\end{array}$ & $\begin{array}{l}\text { Vitamin D dose } \\
(\mu \mathrm{g} / \text { day })\end{array}$ & Nephrocalcinosis \\
\hline 1.1 & $38^{\mathrm{a}}$ & $0.50^{c}$ & No \\
\hline 1.2 & $40^{\mathrm{a}}$ & $0.50^{c}$ & No \\
\hline$\| .1$ & $86^{\mathrm{a}}$ & $1.20^{\mathrm{d}}$ & Yes \\
\hline III.1 & - & - & - \\
\hline IV.1 & $83^{\mathrm{a}}$ & $0.60^{d}$ & No \\
\hline IV.2 & $62^{\mathrm{a}}$ & $1.00^{\mathrm{d}}$ & No \\
\hline V.1 & - & - & - \\
\hline Vl.1 & - & - & - \\
\hline VII.1 & - & - & No \\
\hline VII.2 & - & - & - \\
\hline VIII.3 & $55^{\mathrm{b}}$ & $0.50^{c}$ & Yes \\
\hline VII.4 & $52^{\mathrm{b}}$ & $0.50^{c}$ & Yes \\
\hline VIII.1 & $90^{\mathrm{a}}$ & $1.50^{\mathrm{d}}$ & Yes \\
\hline IX.1 & - & - & - \\
\hline$X .1$ & $40^{\mathrm{a}}$ & $0.25^{d}$ & No \\
\hline XI.1 & $83^{\mathrm{a}}$ & $0.50^{c}$ & No \\
\hline XII.1 & $44^{\mathrm{b}}$ & $0.29^{d}$ & No \\
\hline XIII.1 & $41^{\mathrm{b}}$ & $0.25^{c}$ & Yes \\
\hline XIV.1 & $52^{\mathrm{a}}$ & $1.20^{\mathrm{d}}$ & - \\
\hline$X V .1$ & $41^{\mathrm{b}}$ & $1.00^{c}$ & No \\
\hline XVI.1 & - & - & - \\
\hline XVII.1 & $45^{\mathrm{b}}$ & $0.25^{c}$ & Yes \\
\hline XVIII.1 & - & - & - \\
\hline$X \mid X .1$ & $27^{\mathrm{b}}$ & $0.50^{c}$ & Yes \\
\hline$X X .1$ & $32^{\mathrm{a}}$ & $0.50^{c}$ & No \\
\hline$X X I .1$ & $63^{\mathrm{a}}$ & $1.50^{\mathrm{d}}$ & No \\
\hline XXII.1 & - & - & - \\
\hline XXIII.1 & $65^{\mathrm{a}}$ & $1.10^{\mathrm{d}}$ & No \\
\hline XXIV.1 & - & - & - \\
\hline$X X V .1$ & $38^{\mathrm{a}}$ & $1.40^{c}$ & - \\
\hline XXVI.1 & $58^{\mathrm{b}}$ & $0.25^{c}$ & No \\
\hline XXVII.1 & $48^{\mathrm{b}}$ & $0.60^{d}$ & No \\
\hline$X X V \| I .2$ & - & - & - \\
\hline XXVIII.1 & - & - & - \\
\hline$X X V \mid I I .2$ & - & - & - \\
\hline XXIX.1 & - & $0.10^{d}$ & No \\
\hline$X X X .1$ & - & - & - \\
\hline$X X X .2$ & - & - & Yes \\
\hline XXXI.1 & - & - & - \\
\hline XXXII.1 & - & - & - \\
\hline XXXIII.1 & $49^{\mathrm{b}}$ & $0.75^{c}$ & No \\
\hline XXXIV.1 & - & - & - \\
\hline XXXV.1 & - & - & - \\
\hline$X X X V .2$ & - & - & - \\
\hline XXXVI.1 & - & - & - \\
\hline XXXVII.1 & - & - & - \\
\hline XXXVIII.1 & - & - & - \\
\hline XXXIX.1 & - & - & - \\
\hline
\end{tabular}

Table 5 (continued)

a Phosphate was administered as a solution

b Phosphate was administered as tablets

c Corresponds to 1,25 dihydroxy vitamin D

d Corresponds to 1 hydroxy vitamin $D$

of hyperparathyroidism and nephrocalcinosis. The severity of the disease was similar in males and females and no phenotype-genotype association was found.

\section{Patients and methods}

The RenalTube database including 48 patients, 15 males and 33 females, with the diagnosis of XLH confirmed by defect-of-function mutations found in the PHEX gene was retrospectively reviewed to obtain demographic information and clinical and biochemical manifestations at diagnosis and at the last annual follow-up. Genetic information was confirmed and formatted according to Genome Reference Consortium Human Build 38 patch release 13 (GRCh38.p13) [24]. Variants were analyzed in silico and classified according to recommendations from the consensus of the American College of Medical Genetics and Genomics and the Association for Molecular Pathology [19] as pathogenic, likely pathogenic, benign or likely benign. Results for the age are presented as median and interquartile range (IQR). Other variables are presented for the group as mean $(\mathrm{X}) \pm$ standard error of the mean (SEM). Z score (SDS) of anthropometric values was calculated using Spanish age and sex-matched reference values [25]. Patients with height $\leq 2$ SDS were considered to have longitudinal growth retardation according to World Health Organization standards [26]. Reference values for biochemical parameters were obtained from the laboratory of the Hospital Universitario Central de Asturias (HUCA) [27].

All patients received treatment with phosphate supplementation (dose range of phosphorus element: $27-90 \mathrm{mg} / \mathrm{kg} /$ day at last follow-up) and vitamin D metabolites (dose range: $0.25-1.5 \mu \mathrm{g} /$ day at last followup), according to the criteria and indications given by their physicians (Table 5). Two patients (VII.3 and XV.1) received growth hormone treatment. None of them received burosumab treatment.

Information in RenalTube database was downloaded and formatted to an Excel database. All fields but reasons for consultation and genetic information were multichoice or numeric format.

Chi squared test was used to analyze differences between sex for binary (Yes/No) fields (growth retardation, bone deformities). F-test was used to assess variance equality between sex for anthropometric and biochemical values. T-test was used to analyze differences between sex for anthropometric and biochemical values. Paired 
T-test for means was used to analyze differences between diagnosis and last follow-up for anthropometric and biochemical values. T-test for unequal variances was used to compare age at diagnosis for patients with and without family history of the disease.

Phenotype-genotype correlation was assessed by isolating the most severe phenotypes (lowest serum concentrations, most severe growth retardation, highest levels of alkaline phosphatases) and comparing genetic mutations in these patients looking for big deletions, SNPs with entirely different amino acids or nonsense mutations.

\section{Acknowledgements}

Not applicable.

\section{Authors' contributions}

ER gathered, formattedand analyzed data from patients included in RenalTube database and was a major contributor in writing the manuscript. FS and HG contributed on analyzing data and were major contributors on writing the manuscript. Every other author introduced information of at least 1 patient from this study in RenalTube database. All authors read and approved the final manuscript.

\section{Funding}

This research has been partially funded by Kyowa Kirin Farmacéutica S.L.U., project PI17/01745 from Instituto de Salud Carlos III, Acción Estratégica en Salud 2017-2020 and FEDER funds, Fondo de Investigaciones Sanitarias (FIS), Fundación Nutrición y Crecimiento (FUNDNYC), Instituto de Investigación Sanitaria del Principado de Asturias (ISPA) and Fundación para la Investigación y la Innovación Biosanitaria del Principado de Asturias (FINBA).

\section{Availability of data and materials}

The datasets used and/or analyzed during the current study are available from the corresponding author on reasonable request.

\section{Ethics approval and consent to participate}

Patients information has been processed via RenalTube database where patients signed their consent to participate in scientific studies.

\section{Consent for publication}

Patients or parents signed consent for publication of their data through RenalTube consent form.

\section{Competing interests}

The study has been partially funded by Kyowa Kirin Farmacéutica S.L.U. This company produces the drug Crysvita ${ }^{\circledR}$ (burosumab). Nevertheless, no patients in this study had been or were being treated with burosumab at the time of data collection.

\section{Author details}

${ }^{1}$ Pediatric Research, Medicine Department, University of Oviedo, Oviedo, Spain. ${ }^{2}$ AGC Pediatría, Hospital Universitario Central de Asturias, Oviedo, Spain. ${ }^{3}$ Servicio de Nefrología Pediátrica, Hospital Vall D'Hebron, Universitat Autónoma de Barcelona, Barcelona, Spain. ${ }^{4}$ Servicio Nefrología Pediátrica, IIS Biocruces-Bizkaia, Universidad del País Vasco UPV/EHU, Hospital Universitario Cruces, Barakaldo, Spain. ${ }^{5}$ Unidad de Nefrología Pediátrica, Hospital Virgen del Rocío, Sevilla, Spain. ${ }^{6}$ Servicio Pediatría, Complejo Asistencial Universitario de León, León, Spain. ${ }^{7}$ Servicio Nefrología, Hospital Niño Jesús, Madrid, Spain. ${ }^{8}$ Servicio Pediatría, Hospital Universitario de Santiago de Compostela, Santiago de Compostela, Spain. ${ }^{9}$ Servicio Pediatría, Hospital Universitario Nuestra Señora de Candelaria, Santa Cruz de Tenerife, Spain. ${ }^{10}$ Servicio Pediatría, Complexo Hospitalario Universitario A Coruña (CHUAC), A Coruña, Spain. ${ }^{11}$ Unidad de Nefrología, Hospital General Universitario Santa Lucia, Cartagena, Spain. ${ }^{12}$ Servicio de Pediatría, Hospital Clínico Universitario de Valencia, Valencia, Spain. ${ }^{13}$ Unidad Nefrología Pediátrica, Hospital Universitario Reina Sofia, Córdoba, Spain. ${ }^{14}$ Unidad de Nefrología Pediátrica, Hospital Universitario de Badajoz, Badajoz, Spain. ${ }^{15}$ ServicioNefrología Infantil, Hospital Infantil Gregorio
Marañón, Madrid, Spain. ${ }^{16}$ Unidad de Nefrología Pediátrica, Hospital Virgen de la Salud, Toledo, Spain. ${ }^{17}$ Servicio Nefrología infantile, Hospital Universitario Infantil La Paz, Madrid, Spain. ${ }^{18}$ Servicio Nefrología, Hospital Severo Ochoa, Leganés, Spain. ${ }^{19}$ Servicio Pediatría, Hospital Vega Baja, Orihuela, Spain.

Received: 25 June 2020 Accepted: 3 February 2021

Published online: 27 February 2021

\section{References}

1. Bastepe M, Jüppner H. Inherited hypophosphatemic disorders in children and the evolving mechanisms of phosphate regulation. Rev Endocr Metab Disord. 2008;9(2):171-80.

2. Fuente R, Gil-Peña H, Claramunt-Taberner D, Hernández-Frías O, Fernández-Iglesias Á, Alonso-Durán L, et al. MAPK inhibition and growth hormone: a promising therapy in XLH. FASEB $J$. 2019;33(7):8349-62.

3. Morey M, Castro-Feijóo L, Barreiro J, Cabanas P, Pombo M, Gil M, et al. Genetic diagnosis of X-linked dominant hypophosphatemic rickets in a cohort study: Tubular reabsorption of phosphate and 1,25(OH)2D serum levels are associated with PHEX mutation type. BMC Med Genet. 2011;12(1):116.

4. Zhang C, Zhao Z, Sun Y, Xu L, JiaJue R, Cui L, et al. Clinical and genetic analysis in a large Chinese cohort of patients with X-linked hypophosphatemia. Bone. 2019;121:212-20. https://doi.org/10.1016/j.bone.2019. 01.021.

5. Capelli S, Donghi V, Maruca K, Vezzoli G, Corbetta S, Brandi ML, et al. Clinical and molecular heterogeneity in a large series of patients with hypophosphatemic rickets. Bone. 2015;79:143-9. https://doi.org/10. 1016/j.bone.2015.05.040

6. Francis F, Hennig S, Korn B, Reinhardt R, de Jong P, Poustka A, et al. A gene (PEX) with homologies to endopeptidases is mutated in patients with X-linked hypophosphatemic rickets. Nat Genet. 1995; 1 1 (2):130-6.

7. Fuente R, Gil-Peña H, Claramunt-Taberner D, Hernández O, FernándezIglesias A, Alonso-Durán L, et al. X-linked hypophosphatemia and growth. Rev Endocr Metab Disord. 2017. https://doi.org/10.1007/ s11154-017-9408-1.

8. Gattineni J, Baum M. Genetic disorders of phosphate regulation. Pediatr Nephrol. 2012;27(9):1477-87.

9. Bitzan M, Goodyer PR. Hypophosphatemic rickets. Pediatr Clin North Am. 2019;66(1):179-207. https://doi.org/10.1016/j.pcl.2018.09.004.

10. Carpenter TO, Whyte MP, Imel EA, Boot AM, Högler W, Linglart A, et al. Burosumab therapy in children with $X$-linked hypophosphatemia. $N$ Engl J Med. 2018;378(21):1987-98.

11. Zhukouskaya VV, Rothenbuhler A, Colao A, Di SC, Kamenický P, Trabado $\mathrm{S}$, et al. Increased prevalence of overweight and obesity in children with x-linked hypophosphatemia. Endocr Connect. 2020;9(2):144-53.

12. Emma F, Cappa M, Antoniazzi F, Bianchi ML, Chiodini I, Eller Vainicher C, et al. X-linked hypophosphatemic rickets: An Italian experts' opinion survey. Ital J Pediatr. 2019;45(1):1-7.

13. Cho HY, Lee BH, Kang JH, Ha IS, Cheong HI, Choi Y. A clinical and molecular genetic study of hypophosphatemic rickets in children. Pediatr Res. 2005;58(2):329-33.

14. Endo I, Fukumoto S, Ozono K, Namba N, Inoue D, Okazaki R, et al. Nationwide survey of fibroblast growth factor 23 (FGF23)-related hypophosphatemic diseases in Japan: prevalence, biochemical data and treatment. Endocr J. 2015;62(9):811-6.

15. Ariceta G, Langman CB. Growth in X-linked hypophosphatemic rickets. Eur J Pediatr. 2007;166(4):303-9.

16. Haffner D, Emma F, Eastwood DM, Duplan MB, Bacchetta J, Schnabel D, et al. Clinical practice recommendations for the diagnosis and management of X-linked hypophosphataemia. Nat Rev Nephrol. 2019;15:435-55.

17. Insogna KL, Briot K, Imel EA, Kamenický P, Ruppe MD, Portale AA, et al. A randomized, double-blind, placebo-controlled, phase 3 trial evaluating the efficacy of burosumab, an anti-FGF23 antibody, in adults with X-linked hypophosphatemia: week 24 primary analysis. J Bone Miner Res. 2018;33(8):1383-93.

18. Renaltube. http://renaltube.com/en/ 
19. Richards S, Aziz N, Bale S, Bick D, Das S, Gastier-Foster J, Grody WW, Hegde M, Lyon E, Spector E, Voelkerding KR. Standards and guidelines for the interpretation of sequence variants: a joint consensus recommendation of the american college of medical genetics and genomics and the association for molecular pathology. Genet Med. 2015;17(5):405-24

20. Holm IA, Nelson AE, Robinson BG, Mason RS, Marsh DJ, Cowell CT, et al. Mutational analysis and genotype-phenotype correlation of the PHEX gene in X-linked hypophosphatemic rickets. J Clin Endocrinol Metab. 2001;86(8):3889-99.

21. Rafaelsen S, Johansson S, Ræder H, Bjerknes R. Hereditary hypophosphatemia in Norway: a retrospective population-based study of genotypes, phenotypes, and treatment complications. Eur J Endocrinol. 2016;174(2):125-36.

22. Rothenbuhler A, Schnabel D, Högler W, Linglart A. Diagnosis, treatmentmonitoring and follow-up of children and adolescents with X-linked hypophosphatemia (XLH). Metabolism. 2020;103:153892. https://doi.org/ 10.1016/j.metabol.2019.03.009.
23. Carpenter TO, Imel EA, Holm IA, et al. A clinician's guide to X-linked hypophosphatemia. J Bone Min Res. 2011;26(7):1381-8.

24. Ensembl. https://www.ensembl.org/Homo_sapiens/Gene/Summary? $\mathrm{db}=$ core $; g=$ ENSG00000102174; $\mathrm{r}=\mathrm{X}: 22032325-22251310$

25. Endrocrinoped. http://www.webpediatrica.com/endocrinoped/antro pometria.php

26. WHO. https://www.who.int/nutgrowthdb/about/introduction/en/index5. $\mathrm{html}$

27. Servicio de Bioquímica Clínica (AGC-Laboratorio de Medicina). Hospital Universitario Central de Asturias. Valores bioquímica HUCA. Biblioteca De Pruebas Bioquimicas. 2013.

\section{Publisher's Note}

Springer Nature remains neutral with regard to jurisdictional claims in published maps and institutional affiliations.
Ready to submit your research? Choose BMC and benefit from:

- fast, convenient online submission

- thorough peer review by experienced researchers in your field

- rapid publication on acceptance

- support for research data, including large and complex data types

- gold Open Access which fosters wider collaboration and increased citations

- maximum visibility for your research: over $100 \mathrm{M}$ website views per year

At BMC, research is always in progress.

Learn more biomedcentral.com/submissions 